\title{
eJRIEPS
}

Ejournal de la recherche sur l'intervention en éducation physique et sport

$10 \mid 2006$

Varia

\section{Perceptions d'élèves sur ce qui caractérisent une personne en bonne santé}

Valérie Michaud, Jocelyn Gagnon et Marielle Tousignant

\section{(2) OpenEdition}

Journals

Édition électronique

URL : https://journals.openedition.org/ejrieps/7239

DOI : 10.4000/ejrieps.7239

ISSN : 2105-0821

Éditeur

ELLIADD

Édition imprimée

Pagination : 66-83

Référence électronique

Valérie Michaud, Jocelyn Gagnon et Marielle Tousignant, « Perceptions d'élèves sur ce qui

caractérisent une personne en bonne santé », eJRIEPS [En ligne], 10 | 2006, mis en ligne le 01 juillet 2006, consulté le 29 octobre 2021. URL : http://journals.openedition.org/ejrieps/7239 ; DOI : https:// doi.org/10.4000/ejrieps.7239

a revue eJRIEPS est mise à disposition selon les termes de la Creative Commons Attribution 4.0 International License. 


\section{Perceptions d'élèves sur ce qui caractérisent une personne en bonne santé}

Valérie Michaud, Jocelyn Gagnon, \& Marielle Tousignant

Département d'éducation physique. Université Laval, QC, Canada

Résumé

La plupart des jeunes sont sédentaires, ce qui influence négativement leur condition physique et leur santé. Les conséquences de la sédentarité sur la santé des jeunes ont amené le Ministère de l'Éducation du Québec à intégrer dans les cours d'ÉPS le volet de l'éducation à la santé. Les enseignants d'ÉPS du Québec s'interrogent: Que doivent-ils enseigner spécifiquement en lien avec la santé? Quel est le niveau actuel des jeunes en cette matière? Les élèves ont-ils les connaissances suffisantes pour adopter des habitudes de vie qui contribuent au maintien ou à l'amélioration de leur santé? Cette étude a donc pour but de décrire les perceptions des élèves sur ce qui caractérise une personne en bonne santé afin de vérifier leur niveau de connaissance en cette matière. Elle vise aussi à identifier les personnes qui contribuent à les éduquer au plan de la santé. Soixante-dix-sept élèves $d u$ primaire et 69 élèves du secondaire ont participé à des entrevues de groupe et répondu à un questionnaire. L'analyse des données démontre que les élèves sont en mesure d'identifier les thèmes majeurs qui composent l'univers de la santé ainsi que les principaux comportements à adopter pour être en santé. Cependant, ils sont davantage préoccupés par la dimension physique de la personne en santé au détriment des aspects psychologiques et sociaux. De plus, selon les élèves, les enseignants d'ÉPS ont peu d'impact sur eux, comparativement aux parents qui sont de loin leurs premiers éducateurs à la santé.

Dans nos sociétés occidentales, les jeunes adoptent de plus en plus tôt de mauvaises habitudes de vie (Institut Canadien de la recherche sur la condition physique et le mode de vie (ICRCP), 2001 ; Nolin, Prud'homme, Godin \& Hamel, 2002 ; U.S. Department of Health and Human Services, 1996). La plupart sont sédentaires, ce qui influence négativement leur condition physique et leur santé (ICRCP, 2000). Les effets potentiellement désastreux de la sédentarité chez les jeunes, à court, moyen et long terme, ont donné l'alarme auprès des hauts responsables de ministères de l'éducation de plusieurs États. Ceux-ci ont donc prescrit l'intégration de l'éducation à la santé au cursus de l'éducation physique scolaire. Suivant 
cette logique, le Ministère de l'Éducation du Québec (MÉQ) intégrait en 2001 l'éducation à la santé au cursus scolaire et confiait aux enseignants d'éducation physique la responsabilité d'enseigner ce volet éducatif.

\section{Problématique}

Traditionnellement, l'enseignement de l'éducation physique au Québec était centré sur les actions et sur le développement de l'efficience motrice, le postulat étant que la maîtrise d'habiletés motrices conduirait naturellement les jeunes à pratiquer des activités physiques et sportives (APS) de diverses natures. Les résultats alarmants des études sur les habitudes de vie des jeunes indiquent toutefois qu'il faut aller au-delà de cette efficience si on veut les amener à adopter un mode de vie sain et actif. Dans cette perspective, le nouveau programme d'éducation physique et à la santé (ÉPS) du Québec vise, outre le développement de l'efficience motrice et d'habiletés psychosociales, l'acquisition de connaissances, d'attitudes et de comportements nécessaires à une gestion judicieuse par chacun de sa santé et de son bien-être (MÉQ, 2001). Ainsi, «Adopter un mode de vie sain et actif» est l'une des trois compétences à développer chez les jeunes dans le programme d'ÉPS du Québec.

L'univers de l'éducation à la santé étant très vaste et très peu défini, les enseignants d'ÉPS s'interrogent sur ce qu'ils devront enseigner spécifiquement en lien avec la santé. Quel est le niveau de connaissance actuel des jeunes en cette matière? Savent-ils réellement ce qu'ils doivent faire pour assurer leur santé à court et à long termes? Que signifie être en santé pour les jeunes? À notre connaissance, il existe peu d'études qui ont tenté de mettre en lumière le savoir des jeunes à ce sujet.

1. 1. Pourquoi étudier les perceptions des élèves à l'égard de la santé II semble que mieux circonscrire les connaissances des jeunes sur la santé aurait quelques avantages. Des auteurs mentionnent qu'il est nécessaire de mieux cerner les perceptions des jeunes en cette matière afin d'aider les intervenants à planifier des contenus qui répondent vraiment à leurs besoins (Cogérino, 1999; Ferron, Narring, Cauderay \& Michaud, 1999; Manidi \& Dafflon-Arvanitou, 2000). Coppé et Schoonbroodt (1992) prétendent aussi qu'il est capital de se soucier des représentations sur la santé, notamment parce que ce concept est vague et difficile à cerner, mais aussi parce que chaque personne porte une image différente de ce concept. Selon ces auteurs, s'interroger sur les perceptions et les schèmes de pensée des jeunes est indispensable avant d'entreprendre quoi que ce soit. 
1. 2. Les études portant sur les représentations des jeunes à l'égard de la santé À notre connaissance, quelques études, non récentes, ont été réalisées afin de connaître les perceptions des jeunes sur la santé (Backett \& Alexander, 1991; Harris, 1993; Head, 1987; Murray \& Jarrett, 1985). Les résultats de ces études révèlent qu'ils ont tendance à restreindre le concept de santé à des dimensions physiques, excluant les facteurs sociaux et psychologiques (Backett \& Alexander, 1991; Harris, 1993). Ils n'accordent donc pas beaucoup d'attention aux facteurs psychologiques d'une personne en bonne santé. Ils associent plutôt la santé à des facteurs observables tels que bien manger, ne pas fumer, boire modérément de l'alcool, avoir beaucoup d'énergie, être mince, faire des APS et être performant dans les sports.

1. 3. Les objectifs de cette étude

Cette étude vise à connaître les perceptions des jeunes Québécois sur la santé dans un moment où les enseignants d'ÉPS intègrent progressivement l'éducation à la santé dans leurs cours. De façon plus spécifique, le premier volet de cette étude porte sur la description des perceptions d'élèves sur ce qui caractérise une personne en bonne santé afin de vérifier leur niveau de connaissances dans ce domaine. Le deuxième volet de l'étude vise à identifier les personnes qui, d'après eux, contribuent le plus à les éduquer sur le plan de la santé. Selon Le Goff (2004), on ignore dans quelle mesure ce qu'enseignent les enseignants d'ÉPS est réinvesti par les élèves dans leur vie quotidienne. Ainsi, ce dernier volet permettra de démontrer jusqu'à quel point les élèves sont influencés par leur enseignant d'ÉPS. II s'agira aussi d'expliquer d'où proviennent leurs connaissances sur la santé et qui sont les personnes qui leur ont appris à en prendre soin.

1. 4. Le cadre théorique de l'étude

Le concept de santé a été défini par plusieurs auteurs. Selon Corbin, Lindsey, Welk et Corbin (2004), la santé se définit comme étant un :

"État de bien-être optimal qui contribue à la qualité de vie. La santé représente plus que l'absence de maladie. La santé optimale inclut un haut niveau de bien-être mental, social, émotionnel, spirituel et physique, compte tenu des limites de l'hérédité et des habiletés propres d'une personne » (p. 3).

Nous avons arrêté notre choix sur cette définition car elle fait apparaître le bien-être comme un élément essentiel de la santé. Plusieurs ouvrages utilisent le vocable « mieux-être » en remplacement de l'expression « bien-être ». Ces deux concepts sont donc considérés comme des synonymes par bon nombre d'auteurs. Selon Fahey, Insel et Roth (2003), le mieux-être 
(bien-être) est une version évoluée de la santé et du bien-être physique. Le véritable mieuxêtre découle des décisions concernant les habitudes de vie et pour réussir à y parvenir, il faut s'engager dans un processus dynamique de changement et de croissance dans chacune des six dimensions suivantes: physique, émotive, intellectuelle, spirituelle, interpersonnelle et sociale, environnementale ou planétaire. La façon dont une personne perçoit chaque dimension de son mieux-être influence sa perspective générale de la vie (Corbin, Lindsey, Welk \& Corbin, 2004). Ainsi, le sentiment de mieux-être est différent pour chaque individu. Le cadre théorique présenté à la figure 1 sera utilisé pour l'analyse des résultats afin de faire émerger les caractéristiques d'une personne en bonne santé selon les élèves.

\section{Méthodologie}

\section{1. Participants}

Soixante-dix-sept élèves de $5 e$ et $6 e$ années du primaire (39 garçons et 38 filles) et 69 élèves de secondaire I et II (29 garçons et 40 filles) ont participé à des entrevues de groupe et répondu à un questionnaire. Ces élèves, âgés de 10 à 14 ans, provenaient d'écoles urbaines de la région de Québec. Des entrevues de groupe ont été utilisées pour prélever les perceptions des 146 élèves sur ce qui caractérise une personne en bonne santé et un questionnaire leur a permis d'identifier les personnes qui contribuent le plus à les éduquer sur le plan de la santé.

2. 2. Stratégies de collecte des données

Les entrevues semi-structurées furent réalisées auprès de 11 groupes composés de 10 à 15 élèves de même niveau scolaire. Lors de ces entrevues de groupe, l'animatrice a pris soin de créer un environnement permissif et confortable de façon à ce que chaque personne puisse réagir aux commentaires des autres participants. Elle a aussi invité les moins loquaces à prendre la parole. La question centrale soumise aux élèves était: À quoi reconnaissez-vous qu'une personne est en bonne santé? Cette question devait leur permettre de caractériser une personne en santé afin de vérifier leur niveau de connaissance. Tout au long de l'entrevue, l'animatrice incitait les participants à aller au-delà de leur réponse initiale. Elle leur posait des sous-questions qui les amenaient à préciser leurs propos. Ces entretiens avec les élèves, qui duraient entre 20 et 30 minutes, ont été enregistrés sur des cassettes audio et retranscrits intégralement par la suite. 


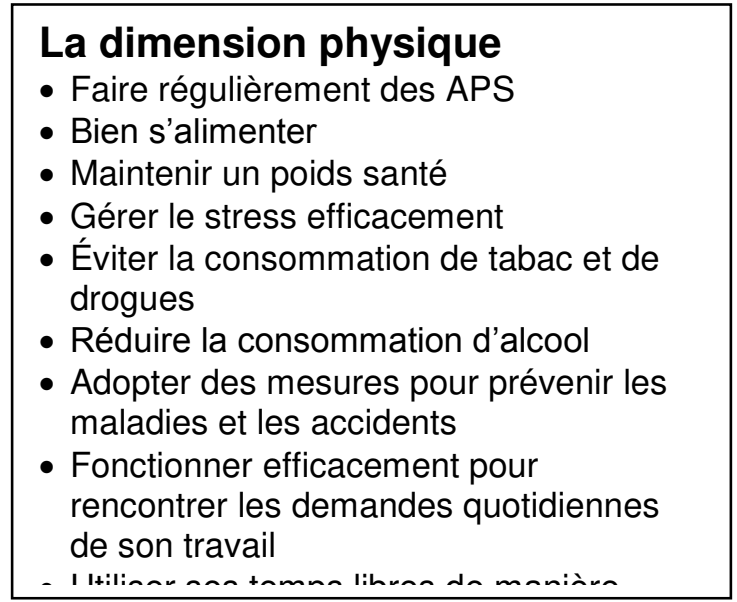

\section{La dimension intellectuelle}

- S'ouvrir aux idées nouvelles

- Faire preuve d'esprit critique

- Avoir le goût d'acquérir de nouvelles compétences

- Faire preuve d'humour

- Faire preuve de créativité

- Faire preuve de curiosité

- Avoir soif d'apprendre

- Prendre plaisir aux nouvelles expériences

- Utiliser à bon escient ses connaissances pour améliorer sa qualité de vie

\section{La dimension émotive}

- Être optimiste

- Avoir confiance en soi et aux autres

- Se respecter

- Avoir une maîtrise de soi

- Établir et maintenir des relations satisfaisantes

- Exprimer ses émotions

- Faire face aux événements en adoptant une attitude positive

- Avoir l'air heureux

\section{La dimension interpersonnelle et sociale}

- Savoir communiquer avec les autres

- Établir des relations d'intimité

- Faire partie d'un réseau social

La dimension
environnementale ou
planétaire
- Être conscient des facteurs
destructeurs de la planète
- Adopter des mesures
susceptibles de nous en
protéger

La dimension spirituelle

- Avoir un ensemble de principes directeurs et de valeurs fondamentales qui donnent un sens et une raison d'être à sa vie

- Être capable d'aimer et faire preuve de compassion

- Pardonner

- Être altruiste

- Être énanoui

Figure 1. Les 6 dimensions du mieux-être (Corbin, Lindsey, Welk \& Corbin, 2004; Fahey, Insel \& Roth, 2003).

Au terme de l'entrevue de groupe, les élèves ont complété individuellement un court questionnaire dans lequel ils devaient identifier les personnes qui leur ont appris à prendre soin de leur santé, puis ils devaient inscrire ce qu'ils avaient appris (Figure 2). Cette procédure a permis de voir dans quelle mesure les élèves sont influencés par leur enseignant d'ÉPS. 
Le questionnaire et le guide d'entrevue ont fait l'objet d'une pré-expérimentation avec quatre groupes d'élèves afin de préciser la terminologie utilisée et vérifier si les questions posées suscitaient des réponses pertinentes.

Niveau scolaire:

Âge:

Sexe:

Nom de ton professeur d'éducation physique:

Pense à un moment précis où tu as appris à prendre soin de ta santé....

Qui est la personne qui t'as enseigné à Qu'est-ce que tu as appris? prendre soin de ta santé?

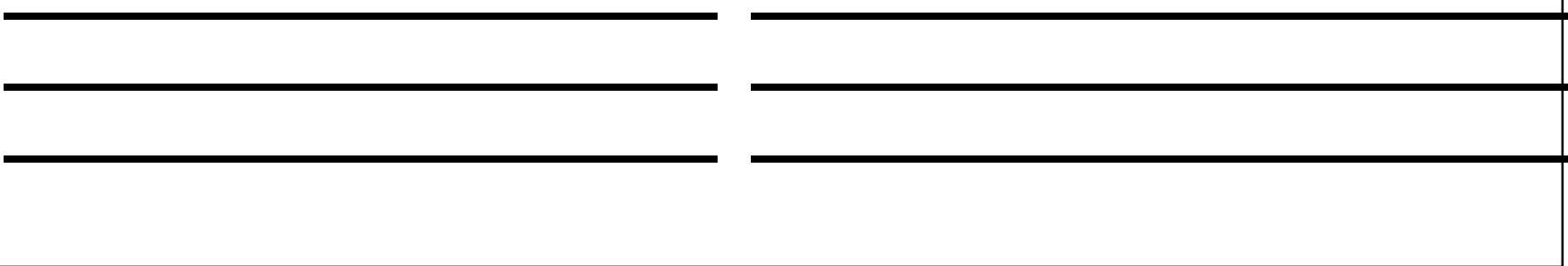

Figure 2 : Questionnaire

\section{3. Stratégie d'analyse des données}

Une stratégie d'analyse de contenu (Deslauriers, 1991; L'Écuyer, 1990) fut réalisée suite à la collecte des données. Les entrevues retranscrites intégralement ont donc été analysées en extrayant des propos des élèves, des caractéristiques d'une personne en bonne santé. Ces caractéristiques ont été regroupées dans des catégories prédéterminées : les six dimensions du mieux-être. Par la suite, un second regroupement a été réalisé afin de mieux faire ressortir les caractéristiques d'une personne en bonne santé dans chacun des indicateurs. Deux chercheures ont effectué de façon indépendante tout ce processus d'analyse de sorte que l'élaboration de la taxonomie fut obtenue sur la base d'un consensus interanalystes. En ce qui concerne le questionnaire, les réponses des élèves ont été regroupées afin de produire une taxonomie des personnes qui contribuent le plus à les éduquer sur le plan de la santé.

\section{Résultats}

3. 1. La répartition des caractéristiques d'une personne en bonne santé selon les six dimensions du mieux-être 
L'analyse des données a permis d'identifier 808 énoncés en lien avec les caractéristiques d'une personne en bonne santé. Les paragraphes suivants présentent les caractéristiques pour chacune des dimensions du mieux-être et des exemples d'énoncés qui illustrent les propos des élèves (Tableau 1).

\section{1. 1. La dimension physique}

\section{Un style de vie active}

Pour les élèves interrogés, une personne en bonne santé est d'abord une personne active (29,2\% des énoncés), peu importe son âge et peu importe les saisons de l'année. Les élèves pensent aussi que les personnes en santé pratiquent beaucoup d'activités d'aérobie telles la natation, la course à pied, la bicyclette, la danse aérobie, le soccer, le hockey, le basket-ball, l'athlétisme, le tennis et le ski de fond ou des activités de préparation physique. À cet égard, les commentaires des plus âgés sont plus précis et révèlent chez eux une meilleure connaissance de certains principes d'entraînement (fréquence, durée) et des composantes d'une séance d'exercices (échauffement, période d'exercices, retour au calme).

Par ailleurs, quelques élèves affirment que l'AP ne se définit pas exclusivement par les loisirs, la pratique sportive et la préparation physique. Ils mentionnent qu'une personne en bonne santé peut aussi faire des AP en accomplissant les gestes utilitaires que lui impose son travail. Ils ajoutent également que certaines personnes profitent d'une activité quotidienne telle que se rendre à l'école ou au travail pour être plus actives physiquement.

Finalement, des élèves reconnaissent qu'une personne en bonne santé occupe ses temps libres en bricolant, en faisant de l'artisanat, en jardinant, en voyageant, en jouant d'un instrument de musique, en cuisinant, etc. Pour eux, la santé est liée à la pratique d'activités, qu'elles soient de nature intellectuelle, sociale, culturelle ou physique.

\section{De bonnes habitudes alimentaires}

Les élèves sont unanimes sur le fait qu'une personne qui prend soin de sa santé se nourrit convenablement $(26,7 \%$ des énoncés). Ils semblent d'ailleurs bien connaître les composantes d'une alimentation saine et équilibrée telles que manger des fruits et légumes en grande quantité, boire beaucoup de lait, consommer des céréales de blé entier, ingérer des aliments qui contiennent des protéines et des vitamines, boire beaucoup d'eau, absorber une alimentation variée. Ils sont aussi conscients qu'il ne faut pas trop manger d'aliments sucrés ou gras. II est à noter que plusieurs d'entre eux considèrent qu'une personne en bonne santé contrôle la quantité de nourriture qu'elle ingère. Cette préoccupation semble associée pour certains, à la nécessité de maintenir un poids corporel satisfaisant. 
Le rejet du tabac et des drogues, une consommation modérée d'alcool et l'utilisation judicieuse de médicaments

Plusieurs sujets affirment qu'un individu en bonne santé ne fume pas, ne consomme pas de drogue et ne prend pas de médicaments. Enfin, pour être en santé, une personne peut boire de l'alcool, mais de façon modérée ( $8,3 \%$ des énoncés).

De saines habitudes d'hygiène

Il est intéressant de constater que les élèves accordent une importance particulière à l'hygiène (6,7\% des énoncés). Ainsi, selon eux, une personne qui est en bonne santé se lave et change de vêtements à tous les jours, prend le temps de se laver après avoir pratiqué une AP et se brosse les dents plusieurs fois par jour. Certains enfants sont également sensibles au fait qu'un individu en bonne santé garde habituellement sa maison propre.

De bonnes habitudes de sommeil

Les élèves estiment qu'une personne en bonne santé dort bien, c'est-à-dire qu'elle s'endort très facilement et ne se réveille pas souvent pendant la nuit. Elle se couche tôt et dort suffisamment longtemps pour permettre une récupération adéquate. Elle dort aussi selon ses besoins, c'est-à-dire "qu'elle va dormir juste assez pour avoir une récupération d'énergie». Par ailleurs, ils considèrent qu'une personne en bonne santé se lève habituellement reposée le matin, sans trop de signes apparents de fatigue (5,2\% des énoncés).

Des comportements pour prévenir la maladie et pour se soigner

Pour certains jeunes, une personne en bonne santé tente d'éviter de contracter des maladies en adoptant des comportements préventifs comme par exemple se prémunir de tous les vaccins. De plus, ils jugent qu'une personne en bonne santé prend le temps de se soigner lorsqu'elle est malade et elle sait comment se soigner (1,9\% des énoncés).

\section{Les attributs physiques d'une personne en bonne santé}

Selon les élèves, l'apparence physique constitue un excellent indicateur de bonne santé (10,9\% des énoncés). Plus spécifiquement, une personne en bonne santé n'est pas obèse, elle a un poids proportionnel à sa taille et est relativement musclée. De même, cette personne soigne son apparence, elle a un beau teint, des dents blanches et elle adopte une bonne posture. La force musculaire, la rapidité et l'endurance sont aussi des qualités que l'on peut retrouver chez des personnes en bonne santé selon le discours des élèves. Plusieurs considèrent également que cette personne est rarement malade et récupère facilement lorsqu'elle l'est. Ils insistent par contre sur le fait que l'absence de maladie n'est pas une 
preuve suffisante de bonne santé. À cet égard, ils rappellent que pour être réellement en santé, il est nécessaire d'être actif physiquement.

\section{1. 2 La dimension émotive}

Seulement $6,3 \%$ des énoncés émis par les élèves font référence à la dimension émotive du mieux-être. Ainsi, l'analyse de leurs propos a permis de constater que certains élèves perçoivent que la bonne santé peut se traduire par l'attitude positive des gens.

\section{Une attitude positive}

Selon eux, une personne en bonne santé s'accepte comme elle est, et est bien dans sa peau. C'est une personne dynamique, de bonne humeur et souriante. Les élèves plus âgés semblent voir le dynamisme sous un oeil différent. En effet, ils décrivent une personne dynamique par l'absence de comportements nonchalants qui, curieusement, sont fréquents chez eux. De plus, des élèves pensent qu'une personne en bonne santé est calme, détient un bon sens de l'humour et ne se plaint pas inutilement. Ils perçoivent également chez cette personne une absence d'agressivité.

\section{1. 3 La dimension spirituelle}

\section{Une générosité envers les autres et une attitude responsable}

Peu de commentaires émis par les élèves se rapportent à la dimension spirituelle du mieux-être (2,6\%). Un petit nombre d'élèves a accordé une attention particulière sur la générosité perçue chez les personnes en bonne santé (1,5\%). Selon eux, cette personne est généreuse, serviable, elle prend soin des gens autour d'elle, elle aime faire plaisir à ses proches et se préoccupe du bien-être d'autrui. Par ailleurs, certains élèves estiment qu'un individu en bonne santé est une personne responsable $(1,1 \%)$, c'est-à-dire qu'elle est consciente que certains de ses comportements peuvent avoir des conséquences sur sa santé. Elle doit donc s'adapter et se comporter en fonction de ce qui est bien pour elle.

\section{1. 4 La dimension intellectuelle}

\section{Une capacité à relever des défis}

Seulement $1,6 \%$ des énoncés concerne la dimension intellectuelle du mieux-être. Les élèves estiment donc qu'une personne en bonne santé persévère face à des difficultés, possède un tempérament de leader et est capable d'entreprendre plusieurs projets.

\section{1. 5 La dimension interpersonnelle et sociale}

\section{De bonnes relations interpersonnelles et sociales}

Pour terminer, un nombre négligeable d'énoncés $(0,6 \%)$ concerne les relations harmonieuses qu'entretiennent, avec les autres, les personnes en bonne santé 


\section{Tableau 1. La répartition des caractéristiques d'une personne en bonne santé selon les six dimensions du mieux-être}

\begin{tabular}{|c|c|c|c|c|}
\hline $\begin{array}{l}\text { Les } \\
\text { dimensions } \\
\text { du mieux- } \\
\text { être }\end{array}$ & Caractéristiques & Exemples d'énoncés émis par les élèves & $\begin{array}{l}\text { Nombre } \\
\text { d'énoncés }\end{array}$ & $\%$ \\
\hline \multirow[t]{7}{*}{$\begin{array}{l}\text { Dimension } \\
\text { physique }\end{array}$} & Un style de vie active & $\begin{array}{l}\text { "Elle est assez âgée et elle fait des travaux comme ramasser } \\
\text { des feuilles. " } \\
\text { "Il fait du jogging à chaque jour, même l'hiver. " } \\
\text { "L'hiver, il joue souvent au hockey et l'été, il joue au soccer et il } \\
\text { va se baigner. " } \\
\text { " Elle fait des sports dans lesquels tu bouges beaucoup, comme } \\
\text { la natation, le tennis, la basket, le hockey. " } \\
\text { "Il marche tout le temps tout au long de la journée parce qu'il } \\
\text { travaille sur un plancher. » } \\
\text { " Elle joue dehors très souvent. » } \\
\text { "Ils font du bricolage, ils travaillent, ils gardent la forme et ne } \\
\text { restent pas écrasés toute la journée. » }\end{array}$ & 236 & 29,2 \\
\hline & $\begin{array}{l}\text { De bonnes habitudes } \\
\text { alimentaires }\end{array}$ & $\begin{array}{l}\text { «Ce n'est pas quelqu'un qui mange du chocolat à chaque jour. II } \\
\text { n'abuse pas. » } \\
\text { « À la place de prendre des chips comme collation, du pop-corn } \\
\text { puis un verre de 7up, elle prend une pomme ou des clémentines. } \\
\text { " II mange les quatre groupes alimentaires. » } \\
\text { « Elle fait attention à son alimentation parce que si elle mange } \\
\text { trop, il faut qu'elle recommence son régime. » }\end{array}$ & 216 & 26,7 \\
\hline & $\begin{array}{l}\text { Le rejet du tabac et } \\
\text { des drogues, une } \\
\text { consommation } \\
\text { modérée d'alcool et } \\
\text { l'utilisation judicieuse } \\
\text { de médicaments }\end{array}$ & $\begin{array}{l}\text { «Des fois à des occasions spéciales, il prend un petit verre de } \\
\text { vin, mais il se contrôle. » } \\
\text { "Elle ne prend pas de médicaments pour dormir. » }\end{array}$ & 67 & 8,3 \\
\hline & $\begin{array}{l}\text { De saines habitudes } \\
\text { d'hygiène }\end{array}$ & $\begin{array}{l}\text { «Il se lave tout le temps les mains. » } \\
\text { «Après avoir fait de l'activité physique, elle prend sa douche. » }\end{array}$ & 54 & 6,7 \\
\hline & $\begin{array}{l}\text { De bonnes habitudes } \\
\text { de sommeil }\end{array}$ & $\begin{array}{l}\text { “Elle dort à peu près } 8 \text { heures par jour. " } \\
\text { "Je ne pense pas qu'une personne en bonne santé se lève à 10- } \\
11 \text { heures à chaque matin, puis qu'elle est encore pochée sur } \\
\text { l'heure du dîner. » } \\
\text { "II n'a pas de misère à s'endormir. " }\end{array}$ & 42 & 5,2 \\
\hline & $\begin{array}{l}\text { Des comportements } \\
\text { pour prévenir la } \\
\text { maladie et pour se } \\
\text { soigner }\end{array}$ & $\begin{array}{l}\text { «Les personnes en santé prennent soin de ne pas attraper de } \\
\text { maladies et de recevoir tous les vaccins. » } \\
\text { "Elle fait attention pour ne pas attraper la grippe. » } \\
\text { "Elle sait se soigner lorsqu'elle est malade. » }\end{array}$ & 15 & 1,9 \\
\hline & $\begin{array}{l}\text { Les attributs } \\
\text { physiques }\end{array}$ & $\begin{array}{l}\text { "Il ne souffre pas d'obésité. " } \\
\text { "Il est assez musclé. " } \\
\text { "Elle n'a pas toujours des poches sous les yeux. » } \\
\text { "Elle se tient droite, elle ne paraît pas toute molle. » } \\
\text { "Elle ne sera pas épuisée après un petit effort physique. C'est } \\
\text { sûr que ce ne sera pas comme si elle était restée inactive, mais } \\
\text { elle va avoir moins de signes de fatigue. » } \\
\text { "Elle est très fière d'elle. Si elle est sale un peu, elle va tout de } \\
\text { suite se changer. Elle n'aime pas être sale. » }\end{array}$ & 88 & 10,9 \\
\hline
\end{tabular}




\begin{tabular}{|c|c|c|c|c|}
\hline $\begin{array}{l}\text { Dimension } \\
\text { émotive }\end{array}$ & Une attitude positive & 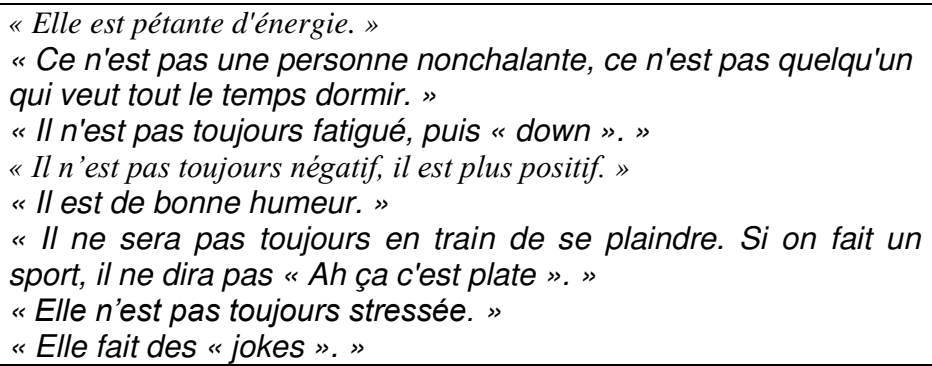 & 51 & 6,3 \\
\hline & & $\begin{array}{ll} & \text { SOUS-TOTAL } \\
\end{array}$ & 51 & 6,3 \\
\hline \multirow[t]{3}{*}{$\begin{array}{l}\text { Dimension } \\
\text { spirituelle }\end{array}$} & $\begin{array}{l}\text { Une générosité } \\
\text { envers les autres }\end{array}$ & $\begin{array}{l}\text { «Elle ne pense pas juste à elle. » } \\
\text { «Elle aide ceux qui ont des difficultés. » } \\
\text { «Elle est serviable et généreuse. » } \\
\text { "Elle aime faire plaisir aux autres. » }\end{array}$ & 12 & 1,5 \\
\hline & $\begin{array}{l}\text { Une attitude } \\
\text { responsable }\end{array}$ & $\begin{array}{l}\text { “D'après moi, la santé c'est une question de conscience... Tu } \\
\text { veux être en santé, alors c'est sûr que tu vas t'organiser pour être } \\
\text { en santé. S'il y a quelqu'un à côté de toi qui fume, tu vas être } \\
\text { conscient qu'il faut que tu t'en ailles pour être en santé. Tu vas } \\
\text { être conscient qu'après quatre barres de chocolat, ça peut être de } \\
\text { l'abus. » }\end{array}$ & 9 & 1,1 \\
\hline & & $\begin{array}{ll} & \text { SOUS-TOTAL } \\
\end{array}$ & 21 & 2,6 \\
\hline \multirow[t]{2}{*}{$\begin{array}{l}\text { Dimension } \\
\text { intellectuelle }\end{array}$} & $\begin{array}{l}\text { Une capacité à relever } \\
\text { des défis }\end{array}$ & $\begin{array}{l}\text { "Elle ne dira pas: c'est trop difficile, je ne le ferai pas. Elle dira: c'est } \\
\text { difficile mais il fait que je l'essaie pareil. » }\end{array}$ & 13 & 1,6 \\
\hline & & $\begin{array}{ll} & \text { SOUS-TOTAL } \\
\end{array}$ & 13 & $\mathbf{1 , 6}$ \\
\hline \multirow[t]{2}{*}{$\begin{array}{l}\text { Dimension } \\
\text { interpersonnelle } \\
\text { et sociale }\end{array}$} & & $\begin{array}{l}\text { "Elle joue avec des amis. » } \\
\text { «Elle a un bon esprit d'équipe. » }\end{array}$ & 5 & 0,6 \\
\hline & & SOUS-TOTAL & 5 & $\mathbf{0 , 6}$ \\
\hline \multirow{2}{*}{$\begin{array}{l}\text { Dimension } \\
\text { environnementa } \\
\text { le ou planétaire }\end{array}$} & & SOUS-TOTAL & $\mathbf{0}$ & $\mathbf{0 , 0}$ \\
\hline & & TOTAL & 808 & 100,0 \\
\hline
\end{tabular}

3. 2 Les personnes qui contribuent à éduquer les élèves sur le plan de la santé Les résultats de l'analyse (Tableau 2), eu égard aux personnes qui éduquent les élèves au plan de la santé, révèlent qu'ils sont surtout influencés par les membres de leur environnement personnel (79\% des réponses). Plus spécifiquement, les parents semblent être les personnes déterminantes en matière d'éducation à la santé $(57 \%)$. Les autres membres de la famille (oncles, tantes, grands-parents, etc.) (16\%) et de l'entourage (voisins, amis) (6\%) sont aussi des personnes relativement influentes. II est à noter que seulement $11 \%$ des réponses émises par les élèves concernent les professionnels de l'éducation, principalement l'enseignant d'ÉPS (8\%) et très peu les autres enseignants (1\%). Quant aux 
entraîneurs sportifs, ils ont été rarement identifiés par les élèves comme des éducateurs à la santé $(2 \%)$.

Par ailleurs, les professionnels de la santé (médecin, dentiste, infirmière, diététiste) sont mentionnés dans seulement $6 \%$ des réponses comme des individus qui permettent de faire des apprentissages relatifs à la santé. De plus, les jeunes se disent peu influencés par les informations transmises par les médias. Seulement $1 \%$ des réponses porte sur des messages écoutés à la télévision. 
Tableau 2. Personnes qui contribuent à éduquer les élèves sur le plan de la santé

\begin{tabular}{|c|c|c|c|}
\hline Catégories & $\begin{array}{l}\text { Nombre de } \\
\text { réponses au } \\
\text { primaire } \\
\text { n (\%) }\end{array}$ & $\begin{array}{l}\text { Nombre de } \\
\text { réponses au } \\
\text { secondaire } \\
\text { n } \quad(\%)\end{array}$ & $\begin{array}{l}\text { TOTAL } \\
\text { n } \%)\end{array}$ \\
\hline \multicolumn{4}{|l|}{ Environnement personnel } \\
\hline $\begin{array}{l}\text { - Les parents } \\
\text { - Les autres membres de la famille } \\
\text { - Les autres personnes de l'entourage }\end{array}$ & $\begin{aligned} 85 & (58 \%) \\
30 & (20 \%) \\
6 & (4 \%)\end{aligned}$ & $\begin{array}{l}104(56 \%) \\
25(13 \%) \\
14(8 \%)\end{array}$ & $\begin{array}{l}189(57 \%) \\
55(16 \%) \\
20(6 \%)\end{array}$ \\
\hline $\begin{array}{ll}\text { TOTAL } \\
\text { The }\end{array}$ & $121(82 \%)$ & $143(77 \%)$ & $264(79 \%)$ \\
\hline \multicolumn{4}{|l|}{ Professionnels de l'éducation } \\
\hline $\begin{array}{l}\text { - L'enseignant d'ÉPS } \\
\text { - L'entraîneur } \\
\text { - Autres enseignants }\end{array}$ & $\begin{array}{cc}7 & (5 \%) \\
1 & (1 \%) \\
0\end{array}$ & $\begin{aligned} 21 & (12 \%) \\
8 & (4 \%) \\
2 & (1 \%)\end{aligned}$ & $\begin{array}{r}28(8 \%) \\
9(2 \%) \\
2(1 \%)\end{array}$ \\
\hline TOTAL & $8(6 \%)$ & $31(17 \%)$ & $39(11 \%)$ \\
\hline $\begin{array}{l}\text { Professionnels de la santé } \\
\text { Médias d'information } \\
\text { Autres }\end{array}$ & $\begin{array}{c}11(7 \%) \\
0 \\
7(5 \%)\end{array}$ & $\begin{array}{ll}8 & (4 \%) \\
2 & (1 \%) \\
2 & (1 \%)\end{array}$ & $\begin{array}{r}19(6 \%) \\
2(1 \%) \\
9(3 \%)\end{array}$ \\
\hline TOTAL & $147(100 \%)$ & $186(100 \%)$ & $333(100 \%)$ \\
\hline
\end{tabular}

\section{Discussion}

4. 1. Les connaissances des jeunes sur la santé

Les résultats de cette étude démontrent que les jeunes ont tendance à restreindre le concept de santé aux dimensions physiques (88,9\%) au détriment des autres dimensions (émotive, spirituelle, intellectuelle, interpersonnelle et sociale et environnementale ou planétaire). Ils font donc davantage référence à des comportements observables pour juger si une personne est en bonne santé. Cette conclusion est d'ailleurs compatible avec celle des études antérieures qui sous-tendent que les jeunes associent peu la santé à des facteurs sociaux et psychologiques mais davantage aux éléments plus perceptibles (Backett \& Alexander, 1991; Harris, 1993). Néanmoins, la quantité et la diversité des caractéristiques d'une personne en bonne santé qui proviennent de l'analyse des propos des élèves de cette étude démontrent qu'ils sont quand même en mesure d'identifier les principaux comportements à adopter pour avoir un mode de vie sain et actif. 
Les études réalisées auprès des jeunes démontrent qu'ils adoptent de plus en plus tôt de mauvaises habitudes de vie et qu'ils sont de moins en moins en bonne condition physique en raison d'une participation limitée à des APS (ICRCP, 2001; Ledent, Cloes, Telama, Almond, Diniz \& Piéron, 1997). Nous émettons l'hypothèse que leurs mauvaises habitudes de vie ne sont sûrement pas liées à un manque de connaissances relatives aux comportements à adopter pour acquérir et maintenir une bonne santé. Cette hypothèse confirme les résultats d'études précédentes qui ont permis d'identifier une contradiction entre les connaissances exprimées par les jeunes en matière de santé et les comportements et attitudes qu'ils privilégient dans leur propre vie (Harris, 1993; Head, 1987; Murray \& Jarrett, 1985).

4. 2. Qui sont les véritables éducateurs à la santé pour les jeunes?

Les parents sont de loin les premiers éducateurs à la santé pour les jeunes. II semble toutefois que les professionnels de l'éducation et de la santé ont un impact plutôt mitigé en matière d'éducation sanitaire. Cet écart entre l'influence des membres de la famille et celle des professionnels de l'éducation et de la santé peut s'expliquer par plusieurs facteurs. D'abord, il faut admettre qu'à titre de premiers responsables de l'éducation de leurs enfants, les parents consacrent généralement beaucoup plus de temps à leur apprendre des notions en lien avec la santé comparativement aux autres intervenants. Ainsi, la majorité des parents supervisent leurs enfants lorsqu'ils adoptent des comportements sanitaires tels que se brosser les dents, bien manger, se laver, jouer dehors, dormir suffisamment et à des heures décentes et régulières. Par ailleurs, il ne faut pas mésestimer l'effet modélisant qu'ils peuvent avoir sur leurs enfants. À titre d'exemple, plusieurs études révèlent que les enfants de parents actifs sont eux-mêmes beaucoup plus actifs que les enfants de parents sédentaires (Hamel, Blanchet \& Martin, 2001; U.S. Department of Health and Human Services, 1996). Enfin, il faut dire que les parents privilégient une éducation axée sur le développement de comportements et d'attitudes favorables en rapport avec divers thèmes de l'éducation à la santé, notamment le sommeil, l'hygiène, la sécurité et l'alimentation, alors que ces mêmes thèmes sont souvent abordés dans une perspective de transmission de connaissances par les professionnels de l'éducation ou de la santé. De plus, cette éducation à la santé transmise par les parents est intégrée dès le plus jeune âge dans les habitudes personnelles des enfants alors qu'à l'école cette éducation est dispensée beaucoup plus tardivement et sporadiquement sous forme de connaissances enseignées collectivement. Finalement, on peut émettre l'hypothèse que le temps consacré aux cours d'ÉPS dans la grille horaire n'est 
probablement pas suffisant pour permettre aux enseignants d'avoir une influence notable chez leurs élèves.

4. 3. Quel rôle peuvent jouer les enseignants d'ÉPS en éducation à la santé?

II est probablement illusoire de penser que les enseignants d'ÉPS puissent être les intervenants les plus influents en éducation à la santé même s'ils figurent parmi les personnes-ressources qui ont pour mission d'aider les jeunes à adopter un mode de vie sain et actif. II convient cependant de s'interroger sur leur impact apparemment limité en cette matière. À la lumière des résultats de cette étude et d'autres études, les jeunes connaissent les comportements à adopter pour être en bonne santé, mais ces connaissances ne semblent pas suffisantes pour qu'ils s'engagent personnellement dans l'adoption de bonnes habitudes de vie. Cette contradiction entre le discours des jeunes et leurs comportements nous amène à questionner l'orientation à donner à l'éducation à la santé en ÉPS. Plusieurs auteurs sont d'accord sur le fait qu'il ne suffit pas de transmettre des connaissances aux jeunes pour susciter des changements dans leurs comportements (Cogérino, 2000; Ledent, Cloes \& Piéron, 1997; McKenzie \& Sallis, 1996). L'enseignement théorique des bienfaits d'un style de vie sain et actif, des méfaits d'un mode de vie malsain, de ce qu'il faut faire et ne pas faire pour être en bonne santé n'est pas une stratégie suffisante puisque les jeunes semblent avoir déjà les connaissances de base sur les facteurs qui favorisent l'amélioration ou le maintien de la santé. Selon l'avis de McKenzie et Sallis (1996), il faut plutôt leur offrir des conditions qui faciliteront leur engagement dans un mode de vie sain et actif.

À cet égard, nous estimons que la portée éducative des enseignants d'ÉPS peut être accrue s'ils orientent leurs interventions sur des aspects ciblés de la santé sur lesquels ils ont plus de contrôle, mettent en place des stratégies d'intervention qui sollicitent l'engagement réel des élèves et s'engagent dans une approche concertée avec d'autres intervenants. Dans cette perspective, une voie qui semble intéressante pour les enseignants d'ÉPS consiste à intégrer progressivement l'éducation à la santé dans leurs cours en utilisant leurs savoirs d'expérience (Pate \& Hohn, 1994). Ainsi, la pratique régulière d'AP devrait être le thème central afin de fonder chez les jeunes une habitude de participation qui contribue au maintien de leur santé (Cogérino, 1999). L'enseignement des contenus liés à la santé ne devrait donc pas être limité à la transmission de connaissances mais être davantage intégré au déroulement habituel des cours d'ÉPS. De façon opérationnelle, les enseignants doivent user 
de stratégies de mise en action telles des contrats d'engagement ${ }^{1}$, des devoirs actifs, des activités de type "Défi »², des programmes d'entraînement, etc., qui visent toutes à favoriser l'augmentation du niveau de pratique d'AP des élèves ou une modification tangible d'habitudes de vie plus ou moins saines. Ces stratégies mises en place dans les cours d'ÉPS sollicitent aussi l'engagement des élèves à l'extérieur des cours prévus à l'horaire scolaire afin de les responsabiliser face à leur pratique personnelle d'APS.

II semble également que les enseignants d'ÉPS qui se donnent comme mandat d'influencer le mode de vie des élèves auraient avantage à entreprendre des actions concertées avec différents intervenants tels les titulaires de classe, les spécialistes des autres matières, l'infirmière, le travailleur social et les parents. En mettant en place des projets interdisciplinaires ou des projets avec la famille, ils pourraient contribuer à enrichir l'environnement scolaire. À cet effet, l'étude de Gauthier (2005) démontre qu'une des conditions essentielles à la réussite de projets visant à amener les élèves vers un mode de vie actif est d'avoir le soutien et la collaboration d'intervenants qui ont une influence directe sur les élèves tels que les titulaires de classe et les parents. Coppé et Schoonbroodt (1992) précisent aussi qu'il ne convient pas que l'éducation à la santé soit une activité ponctuelle, isolée et déconnectée du contexte global du programme de l'élève. L'intégration de l'éducation à la santé dans le curriculum scolaire suppose un corps professoral motivé et préparé ainsi qu'une participation de plusieurs intervenants de l'école (Coppé \& Schoonbroodt, 1992; Manidi \& Dafflon-Arvanitou, 2000).

\footnotetext{
1 Stratégie d'enseignement personnalisée dans laquelle l'élève doit choisir une habitude de vie à améliorer, déterminer un objectif précis à atteindre et des moyens d'action pour y arriver. II doit, tout au long du contrat, noter ses résultats périodiquement, faire des bilans et tirer des conclusions sur les actions à entreprendre pour le futur.

2 Stratégie qui consiste pour l'élève à adopter pendant un certain temps une habitude de vie déterminée par l'enseignant d'ÉPS (pratique régulière d'APS, alimentation, sommeil, consommation de tabac, hygiène, etc.). Tout au long du défi, l'élève doit comptabiliser sa progression vers l'atteinte du défi en accumulant des points, des minutes d'AP, des kilomètres, etc. Au terme de l'activité, il doit avoir complété en totalité le défi qui lui était proposé.
} 


\section{Bibliographie}

Backett, K. \& Alexander, H. (1991). Talking to young children about health: Methods and findings. Health Education Journal, 50(1), 34-38.

Cogérino, G. (1999). Apprendre à gérer sa vie physique. Paris: Presses Universitaires de France.

Cogérino, G. (2000). Curriculum en éducation physique et éducation à la santé : débats autour d'une difficile intégration. STAPS, 53, 79-90.

Coppé, M. \& Schoonbroodt, C. (1992). Guide pratique d'éducation pour la santé. Bruxelles: De Boeck Université.

Corbin, C.B., Lindsey, R. Welk, G.J. \& Corbin, W.R. (2004). Actif et en santé. Québec : Les Éditions Reynald Goulet inc.

Deslauriers, J.P. (1991). Recherche qualitative. Guide pratique. Montréal : Chenelière/McGraw-Hill.

Fahey, T.D., Insel, P.M. \& Roth, W.T. (2003). En forme et en santé. Mont-Royal : Modulo Éditeur.

Ferron, C., Narring, F., Cauderay, M. \& Michaud, P.A. (1999). Sport activity in adolescence: associations with health perceptions and experimental behaviours. Health Education Research, 14(2), 225-233.

Gauthier, K. (2005). Stratégies d'éducation à la santé qui visent à rendre les élèves plus actifs. Mémoire de maîtrise non publié, Département d'éducation physique, Université Laval, Québec.

Hamel, M., Blanchet, L. \& Martin, C. (2001). Nous serons bien mieux. Les déterminants de la santé et du bien-être des enfants d'âge scolaire. Québec: Les publications du Québec.

Harris, J. (1993). Young people's perceptions of health, fitness and exercise. British Journal of Physical Education, 13, Research supplement, 5-9.

Head, M.J. (1987). Health beliefs in adolescence : Perceptions and control. Health Education Journal, 46(3), 100-103.

ICRCP (2001). Sondage indicateur de l'activité physique. Santé Canada. Gouvernement du Canada. 
ICRCP (2000). Sondage indicateur de l'activité physique. Santé Canada. Gouvernement du Canada.

L'Écuyer, R. (1990). Méthodologie de l'analyse développementale de contenu. Méthode GPS et concept de soi. Sillery : Presses de l'Université du Québec.

Ledent, M., Cloes, M. \& Piéron, M. (1997). Les jeunes, leur activité physique et leurs perceptions de la santé, de la forme, des capacités athlétiques et de l'apparence. Sport, 159/160, 90-95.

Ledent, M., Cloes, M., Telama, R., Almond, L., Diniz, J. \& Piéron, M. (1997). Participation des jeunes Européens aux activités physiques et sportives. Sport, 159/160, 61-71.

Le Goff, V. (2004). Impact de l'éducation physique scolaire dans la vie courante d'élèves du secondaire. Mémoire de maîtrise non publié, Département des Sciences de l'activité physique, Université du Québec à Trois-Rivières, Trois-Rivières.

Manidi, M.J. \& Dafflon-Arvanitou, I. (2000). Activité physique et santé. Apports des sciences humaines et sociales. Éducation à la santé par l'activité physique. Paris : Masson.

McKenzie, T.L. \& Sallis, J.F (1996). Physical activity, fitness, and health-related physical education. Dans: S.J. Silverman \& C.D. Ennis (Eds.), Student learning in physical education: Applying research to enhance instruction. (pp. 223-246). Champaign, IL: Human Kinetics.

Ministère de l'Éducation du Québec. (2001). Programme de formation de l'école québécoise. Québec : Gouvernement du Québec.

Murray, M. \& Jarrett, L. (1985). Young people's perceptions of health, illness and smoking. Health Education Journal, 44(1), 18-22.

Nolin, B., Prud'homme, D., Godin, G. \& Hamel, D. (2002). Enquête québécoise sur l'activité physique et la santé. Institut de la statistique du Québec, Institut national de santé publique du Québec et Kino-Québec.

Pate, R.R. \& Hohn, R.C. (1994). A contemporary mission for physical education. Dans: R.R. Pate \& R.C. Hohn (Eds.), Health and fitness through physical education. (pp. 1-8). Champaign, Illinois: Human kinetics.

U.S. Department of Health and Human Services. (1996). Physical Activity and Health: A report of the Surgeon General. Atlanta, GA: U.S. Department of Health and Human Services, Centers for Disease Control and Prevention, National Center for Chronic Disease Prevention and Health Promotion. 